\title{
FIRE DESIGN IN SAFETY ENGINEERING: LIKELY FIRE CURVE FOR PEOPLE'S SAFETY
}

\author{
MARA LOMBARDI ${ }^{1}$, GIULIANO ROSSI ${ }^{1}$, NICOLÒ SCIARRETTA ${ }^{1}$, \\ LUCA GROSSI $^{2} \&$ NINO ORANGES ${ }^{1}$ \\ ${ }^{1}$ Department of Chemical Engineering Materials Environment, Sapienza University of Rome, Italy \\ ${ }^{2}$ Department of Astronautical, Electrical and Energy Engineering, Sapienza University of Rome, Italy
}

\begin{abstract}
The present study analyses fire design settings according to Fire Safety Engineering (FSE) for the simulation of fire in civil activities and compares these simulations developed using natural and analytic fire curves. The simulated Heat Rate Release (HRR) curve, appropriately linearized, allows for the estimation of a Likely Fire Curve (LFC). The analytic curves have been introduced for the purpose of evaluating the strength and integrity of the structure, and the adoption of these curves in the fire safety engineering was made following the assumption that the phenomena of major intensity ensure the safe approach of fire design. This argument describes the method adopted for determining a likely fire model that guarantees a greater adherence of the virtualized phenomenon with respect to the potential event. The study showed that the analytic curve, adopted in order to verify the structural strength, in the beginning phases of fire produces fields of temperature and toxic concentrations lower than those obtained by simulation of the Likely Fire Curve. The assumption of the Likely Fire Curve model safeguards exposed people during self-rescue and emergency procedure. The programs used since 2011 for the simulation are FDS (Fire Dynamic Simulator v. 5.4.3) and Smokeview (5.4.8). Comparative analysis was developed using thermo-fluid dynamic parameters (temperature and heat release rate) relevant to the safety of the exposed persons; the case study focuses on children and employees of the nursery. The main result shows that the safety criterion, implicitly included in the analytical fire curves - normally used for fire resistance - doesn't have the same applicability of a performance based approach on safety evaluation involving people. This paper shows that the Likely Fire Curve assumption involves a thermo-chemical stress more relevant to assessing the safety of exposed people.
\end{abstract}

Keywords: Analytic Fire Curve (AFC), Fire Safety Engineering (FSE), Likely Fire Curve (LFC), nursery safety.

\section{INTRODUCTION}

The objective of fire safety applied to nursery-based facilities requires a punctual examination of the technical code framework in order to guarantee the correspondence of the requisite norms with their efficacy regarding the need for saving the children, a category particularly vulnerable and in need of active protection.

Inside the technical fire safety standards that are adopted in Italy (Annex I to D.P.R. 151/2011) [1], nurseries with over 30 persons present are taken into account among the activities subject to firefighting inspections.

The regulatory references that were adopted before the issuance of the D.M. 16/07/2014 [2], consisted of:

1. The guideline of the Regional Directorate of Friuli Venezia Giulia or the provincial command of Palermo for fire safety aspects;

2. For architectural, urban and health aspects, the D.M. 18/12/1975 or newer municipal laws were taken into account. 
The technical specifications contained in the standard regulation formally solves the problem of legislative alignment, but the existing laws can neither tackle the critical conditions resulting from the psychophysical characteristics of the users, nor those linked to the structural or organizational features of historic buildings or urban areas with particular features.

This deficiency can be overcome by adopting performance-based fire safety design methods applied in Italy since 2007 (D.M. 09 May 2007) in the framework of fire prevention procedures [3].

With this article is proposed a modelling criterion for the determination of thermo-fluid dynamics parameters. Those parameters are evaluated on the basis of models that relate to the realistic conditions of the activity. The scope of the research of those parameters is relevant to the safety of the exposed nurseries and the comparison with their respective natural and nominal fire curves that are frequently used.

The decisive factors for defining the level of fire safety and emergency planning in these activities are influenced by the peculiarities that characterize the users: children between 0 and 3 years, who are not self-sufficient and are in need of assistance under ordinary conditions and even more in critical condition.

The study space analyzed in FDS5 for the simulation of a fire [4], [5], was limited to the multi-task room and the fire event was simulated for a duration of just over 15 minutes after the start of the fire. This interval of time was used considering the fact that the assessment of the hazard flow to the exposed subjects is relevant only in this first phase.

There were three environments built in FDS with typical kindergarten furnishings [6], but differentiated by geometric dimensions, ventilation openings and quantity of fuel.

We performed four simulations for each of the three environments: in the first, the parameters of the fire were derived from the simulation characterized by the analytic HRR curve, while in the other three, the HRR function was obtained afterwards by simulating the realistic furniture in which the minimum effective trigger system is characterized by two values that are representative of the thermal flow:

- temperature of the radiant surface,

- activation time of the radiant surface.

From the trend of simulated HRR curves, opportunely linearized, were obtained nine Likely Fire Curves (LFC).

A comparison was carried out between the fire parameters obtained from the heat rate release $[\mathrm{kW}-\mathrm{t}]$ the derived from the results of the nine simulations, with the corresponding analytical fire curves that constitute the fire engineering guidelines.

Historically the most widely used natural curves were introduced in order to verify structural strength, and by analogy, these models were also adopted in the fire risk assessment, assuming that the intensity of the phenomenon that it includes is a guarantee for the choice of fire design.

Instead this study points out that the analytic curve generates fields of temperatures below those obtained by the complete simulation of natural fire in the rising and growth phase, that is to say the phase that require the activation and management of exodus even without the firefighters' action.

Our result shows that using only the criteria from analytical fire curves fails to fully represent a generalizable method that a standardized criterion should contain. 
Under this consideration, it is essential to adopt computational dynamic fluid models to design the heat release rate $\left(\mathrm{HRR}_{\mathrm{LFC}}\right)$ and to ensure greater overlap to scenarios that may potentially occur.

In the context of activities that include firefighting inspections, the adoption of ad hoc thermo-chemical models represents the starting point for: defining fire scenarios, assessing the safety of exposed persons, designing appropriate management procedures and protection to reach a safe place while overcoming levels of lethality.

This constraint verifies the ASET $>$ RSET (Available Safe Egress Time / Required Safe Egress Time) concept and is summarized [7]; the combination of thermal stress and toxic gas concentration represents the variable that most influences the available time to evacuate.

The software programs used for the simulation are FDS (Fire Dynamic Simulator) v. 5.4.3 and Smokeview 5.4.8 both produced by the National Institute of Standards and Technology (NIST). FDS (Fire Dynamic Simulator) was developed by the National Institute of Standards and Technology (NIST) Fire Research Division - Building and Fire Research Laboratory (BFRL), which has been adapted to over 25 years of validation at different scales Resolution of fire scenarios [8], [9].

\section{SIMULATION LAYOUT}

The three environments simulated reproduce the typical multi-task nursery area; in the picture, it is possible to see an example.

The rooms are characterized by square plans, different areas and volumes, but the internal height is constant $(3.2[\mathrm{~m}])$, and the thickness of the walls is $0.2[\mathrm{~m}]$.

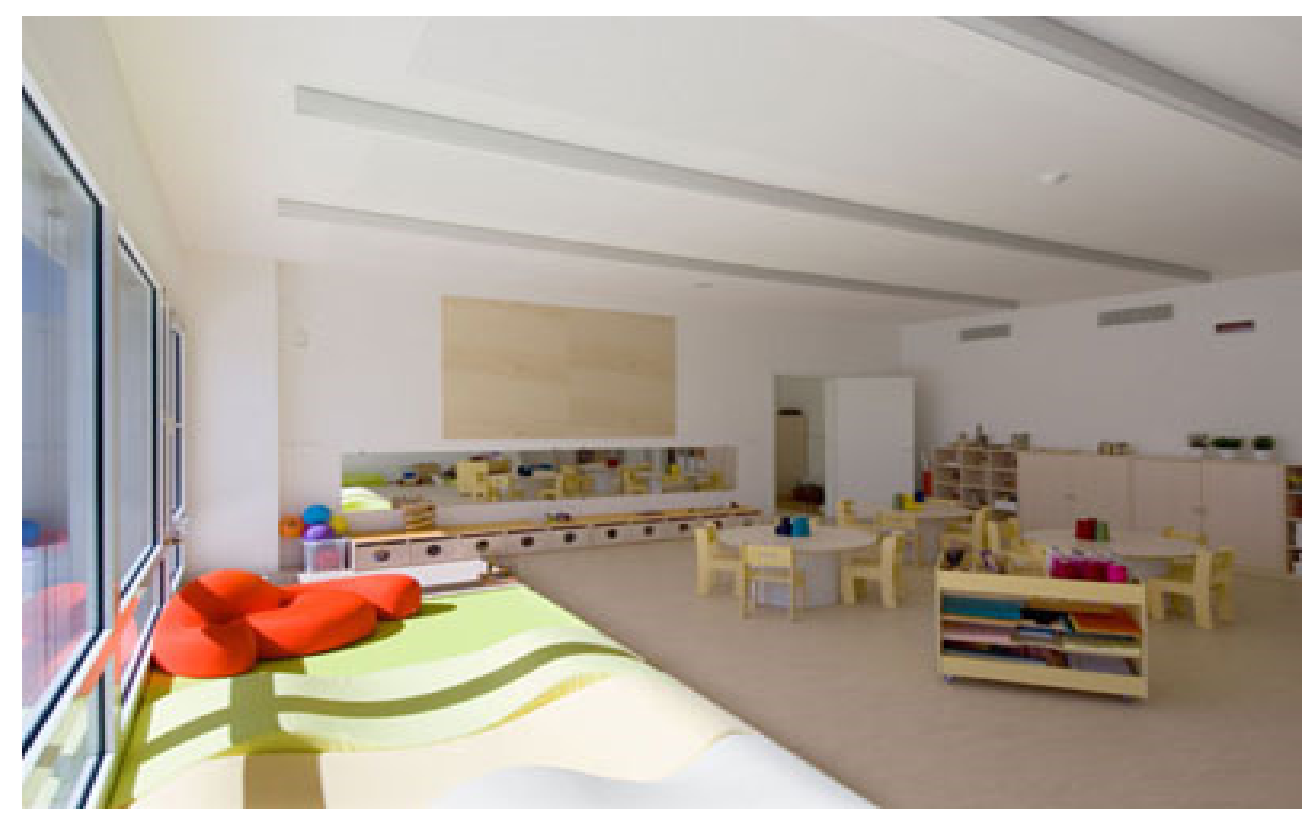

Figure 1: An example of a typical multi-task area. (Source: Google Images.) 
Table 1: Layout of simulated area - (Areas and volumes refer to the inside dimensions of the room).

\begin{tabular}{|c|c|c|c|}
\hline & Small Room & Middle Room & Large Room \\
\hline $\mathrm{n}^{\circ}$ mesh $[5 \times 5 \times 5 \mathrm{~cm}]$ & 663552 & 1179648 & 1620000 \\
\hline Area $\left[\mathrm{m}^{2}\right]$ & $14.82(3.85 \times 3.85)$ & $29.70(5.45 \times 5.45)$ & $44.89(6.70 \times 6.70)$ \\
\hline Volume $\left[\mathrm{m}^{3}\right]$ & 47.43 & 95.04 & 143.64 \\
\hline Door $\left[\mathrm{n}^{\circ}\right]$ & 1 & 1 & 1 \\
\hline Windows $\left[\mathrm{n}^{\circ}\right]$ & 1 & 2 & 3 \\
\hline Sup. Vent. $\left[\mathrm{m}^{2}\right]$ & 2.96 & 4.16 & 5.36 \\
\hline$\%$ Sup. trasparente & $18,6 \%$ & $14.00 \%$ & $11.94 \%$ \\
\hline
\end{tabular}

Table 2: Quantities of combustible furniture.

\begin{tabular}{|c|c|c|c|}
\hline & Small Room & Middle Room & Large Room \\
\hline Mattress in polyurethane $[\mathrm{kg}]$ & 149,76 & 299,52 & 449,28 \\
\hline Table and chairs in wood [Kg] & 39,75 & 78,75 & 100.5 \\
\hline Furniture in wood [Kg] & 32,625 & 62,625 & 92,43 \\
\hline Curtains [Kg] & 0,506 & 1,01 & 2,37 \\
\hline Paper and cardboard [Kg] & 6,03 & 10,95 & 16,2 \\
\hline Plastic bottles [Kg] & 0,9375 & 1,87 & 1,87 \\
\hline Kg [tot] & 229,60 & 454,73 & 662,65 \\
\hline
\end{tabular}

Table 3: Lower heating value of furniture.

\begin{tabular}{|l|c|}
\hline & Lower heating value \\
\hline Polyurethane $[\mathrm{MJ} / \mathrm{Kg}]$ & 41,86 \\
\hline Wood[MJ/Kg] & 15,00 \\
\hline Curtains $[\mathrm{MJ} / \mathrm{Kg}]$ & 30,00 \\
\hline Paper $[\mathrm{MJ} / \mathrm{Kg}]$ & 14,00 \\
\hline Plastic bottles $[\mathrm{MJ} / \mathrm{Kg}]$ & 25,00 \\
\hline
\end{tabular}

The calculation was conducted assuming the windows and the door completely open.

The rooms were furnished in a specific way and based on the information found during some inspections carried out in a real structure.

\section{ANALYTICAL FIRE DESIGN}

The mathematical model adopted to evaluate the fundamental parameters is based on the analytical method indicated in NFPA 555 (that includes the Thomas method for evaluating the HRR of the flashover, Eurocode 1 method for evaluating the maximum HRR value and geometric considerations) and it characterizes the three heat release rate analytical curves [9] [10].

Based on this model, though with necessary simplifications, the analytical fire curve is elaborated on the real fire conditions. Those conditions, with reference to combustible material and opening factors, determine the typical fire evolution time (rise, growth, full development, decay) (Fig. 2). 
The fire load due to different combustible materials was calculated using the following formula:

$$
q_{f}=\sum_{i=1}^{n} g_{i} \cdot H_{i}[\mathrm{MJ}]
$$

where $g_{i}$ represents the mass of the fuel [kg], while Hi represents the lower calorific value of the combustible material $[\mathrm{MJ} / \mathrm{kg}]$;

The chart below shows the formulas and parameters for the determination of the heat release rate curve in accordance with the instructions contained in the Thomas method in NFPA 555 [11], NFPA 72 and 92B and in Eurocode UNI EN 1991-1-2 [13].

The HRR curve [kW-t] for school-related environments has a rising phase characterized by the mean growth factor of the fire: $\alpha=0.01111\left[\mathrm{~kJ} / \mathrm{s}^{3}\right][13],[15]$. The data pertinent to the phenomenon are summarized in Table 4, while Fig. 3 shows the trends of the HRR(t) characterized by the ignition and the growth phases. Conventionally the full development phases stop when $70 \%$ of the available fuel is consumed; at this point the decay phase begins. Table 4 shows that in all three analyzed cases, the opening factor is lower than the conventional value of 0.07 [m 1.5], generating a ventilation controlled fires.

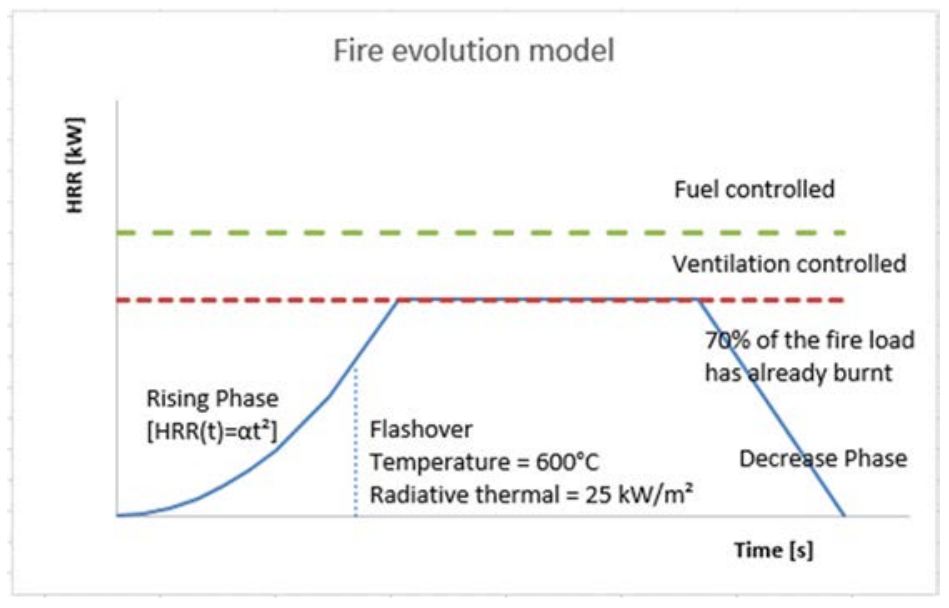

Figure 2: Fire evolution model [16].

Table 4: Characteristic parameters for the three simulation conditions.

\begin{tabular}{|c|c|c|c|}
\hline & Small Room & Middle Room & Large Room \\
\hline Fire Load [MJ] & $7,477.62$ & $14,889.01$ & $22,045.46$ \\
\hline Opening factor & 0.0502 & 0.0410 & 0.0377 \\
\hline HRR $_{\text {FLASHOVER }}[\mathrm{MW}]$ & 2.51 & 4.18 & 12.71 \\
\hline HRRMAX $[\mathrm{MW}]$ & 7.07 & 11.35 & 15.39 \\
\hline tFLASHOVER [s] & 476.02 & 613.55 & 1069.66 \\
\hline$t_{\operatorname{MAX}(\mathrm{A})}[\mathrm{s}]$ & 798.12 & 1011.03 & 1177.25 \\
\hline $\mathrm{t}_{\mathrm{MAX}(\mathrm{B})}[\mathrm{s}]$ & 1273.9 & 1591.69 & 1786.99 \\
\hline t tFINAL [s] & 1909.8 & 2378.24 & 2645.95 \\
\hline
\end{tabular}




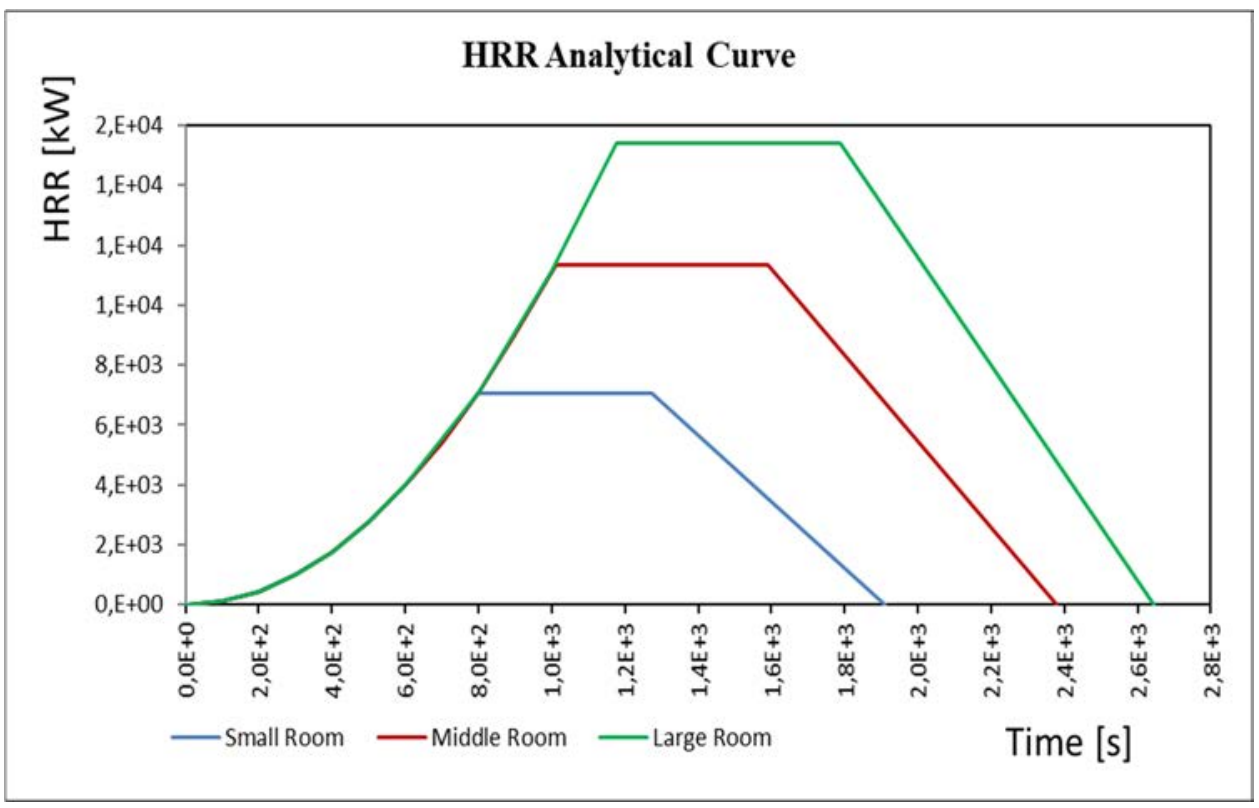

Figure 3: Heat release rate curve $\operatorname{HRR}(\mathrm{t})$.

The fire simulation was developed by inserting a "burner" into the volume that was set into the previously elaborated HRR function.

The temperature trends obtained from the simulations are shown below and obtained with a device positioned on the ceiling barycenter.

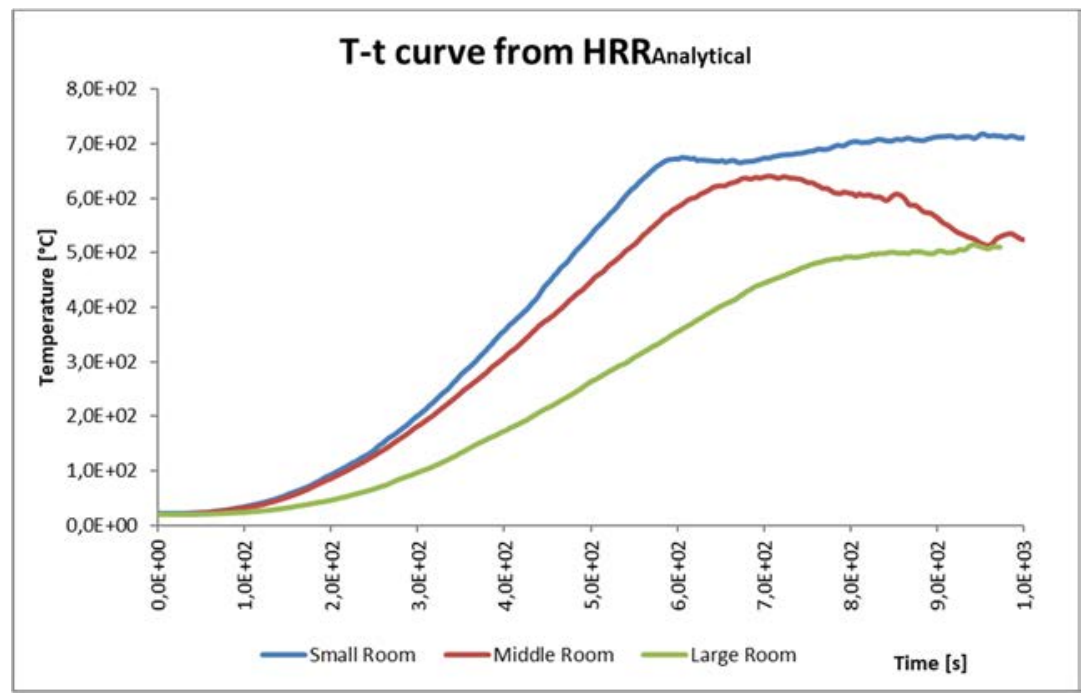

Figure 4: Evolution of temperature-time adopting the HRR analytical curve. 
Growth phases show a pseudolinear pattern until the maximum temperature is reached, but the comparison shows that the angular coefficient decreases as the compartment area increases.

\section{LIKELY FIRE CURVE "HRR LFC"}

With the purpose of comparing the obtained results, the three environments described in paragraph 2, that present the same geometric pattern and the same contour conditions, were adopted in the evaluation of the analytical curve.

The trigger was obtained by inserting a radiant surface of $0.1 \mathrm{~m} \times 0.1 \mathrm{~m}$ underneath one of the polyurethane beds; said device is characterized by a predetermined temperature and operating time.

The characteristics of the primer were obtained through a series of simulations designed to obtain the minimum effective temperature to ensure a long-lasting combustion process even after the primer system is exhausted, until it reaches the flashover condition. In-depth analysis on the trigger system aims to reproduce the effects of a natural trigger (for example, triggers due to short-circuited electrical devices).

From the analysis described above, the minimum temperature set for the activation duration of the primer was 150, 300 and 600 seconds analogous to the technical reference used to estimate the " $\alpha$ " growth factor [11].

The analyzed trigger system returns the three temperature-time functions for each of the studied environments. Thus, it allows the determination of the lower limit for the selected parameter combinations to reach the flashover. Under this limit, the combustion process does not reach the flashover condition; it means that the use of all the combinations of temperature and time in the underlying area function cannot influence quantitative risk assessments.

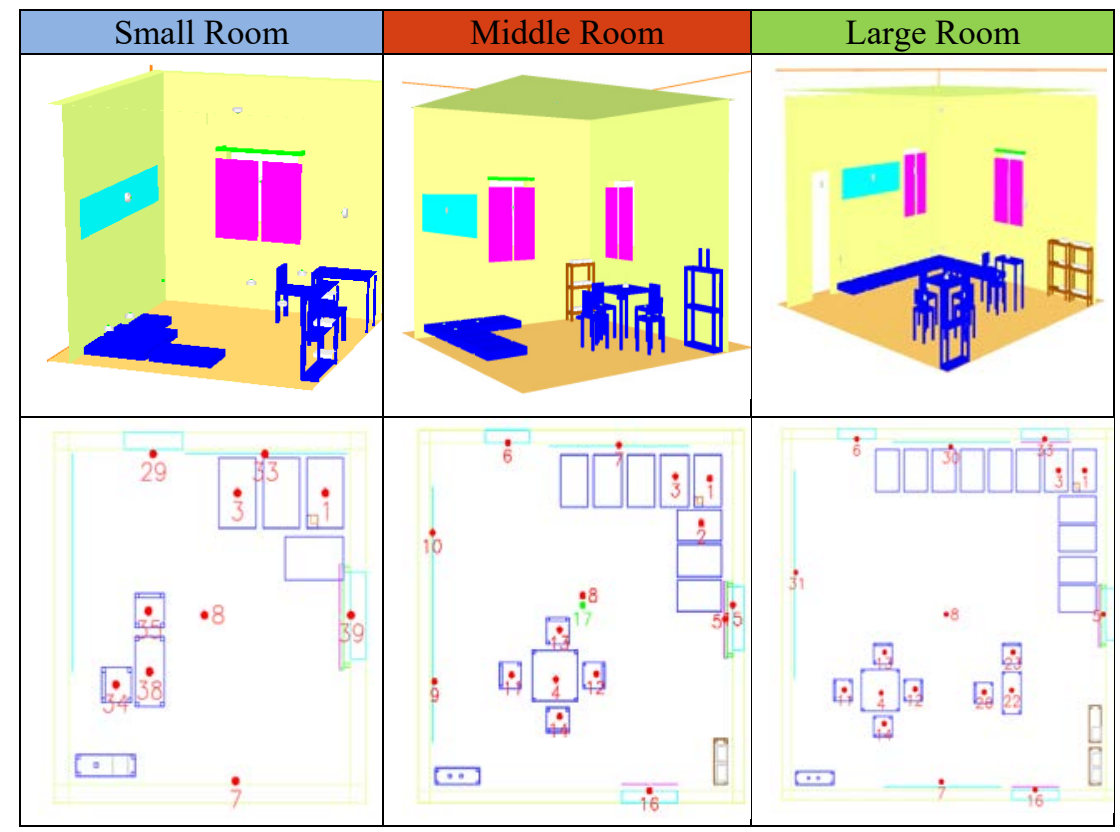

Figure 5: 3D View from smoke view. Example of furnishings in simulated room, arrangement of the furniture plan, windows and measuring device. 


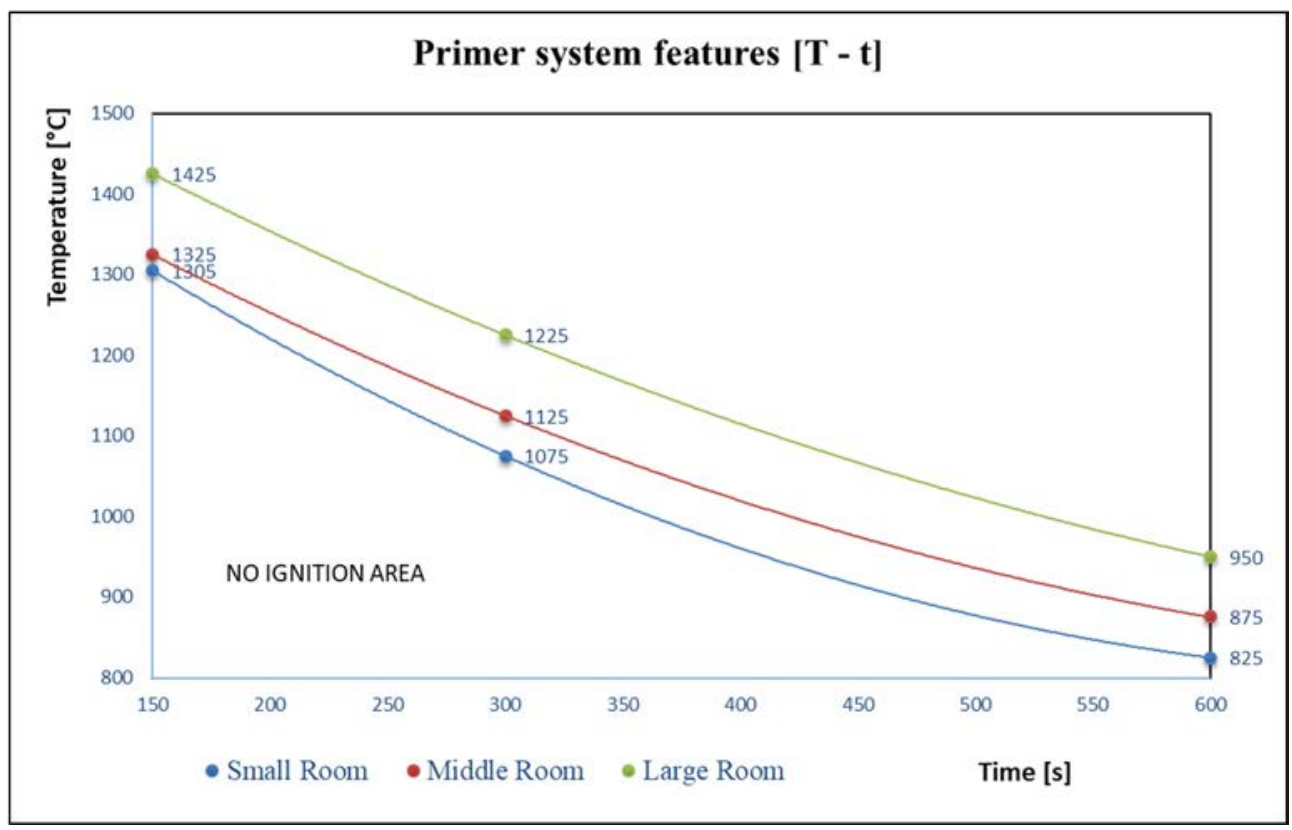

Figure 6: Temperature-time function of the simulation rooms. The boundary lines represent the parameter combinations that the combustion process can or cannot reach the flashover condition.

Table 5: the minimum effective temperature in input for HRR likely curve.

\begin{tabular}{|c|c|c|c|}
\hline Time $[\mathrm{s}]$ & Small Room $\left[{ }^{\circ} \mathrm{C}\right]$ & Middle Room $\left[{ }^{\circ} \mathrm{C}\right]$ & Large Room $\left[{ }^{\circ} \mathrm{C}\right]$ \\
\hline 150 & 1305 & 1325 & 1425 \\
\hline 300 & 1075 & 1125 & 1225 \\
\hline 600 & 825 & 875 & 950 \\
\hline
\end{tabular}

Table 6: temperature-time functions for input simulation parameter.

\begin{tabular}{|c|c|}
\hline Small Room & $\mathrm{T}=0,0016 \mathrm{t}^{2}-2,23 \mathrm{t}+1605$ \\
\hline Middle Room & $\mathrm{T}=0,0011 \mathrm{t}^{2}-1,83 \mathrm{t}+1575$ \\
\hline Large Room & $\mathrm{T}=0,0009 \mathrm{t}^{2}-1,75 \mathrm{t}+1666,7$ \\
\hline
\end{tabular}




\section{DISCUSSION OF RESULTS}

The fire simulation developed in the described conditions has returned the following set of results:

1. $\quad$ HRR-t simulated (Fig. 7 - Fig. 8 - Fig 9);

2. Evolution T-t curve in the simulated area (Fig. 10).

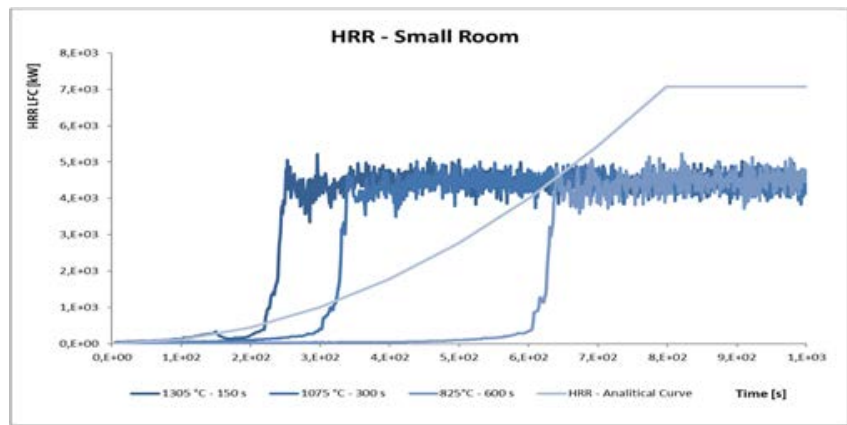

Figure 7: HRR(t) likely curve simulated in small room with FDS5.

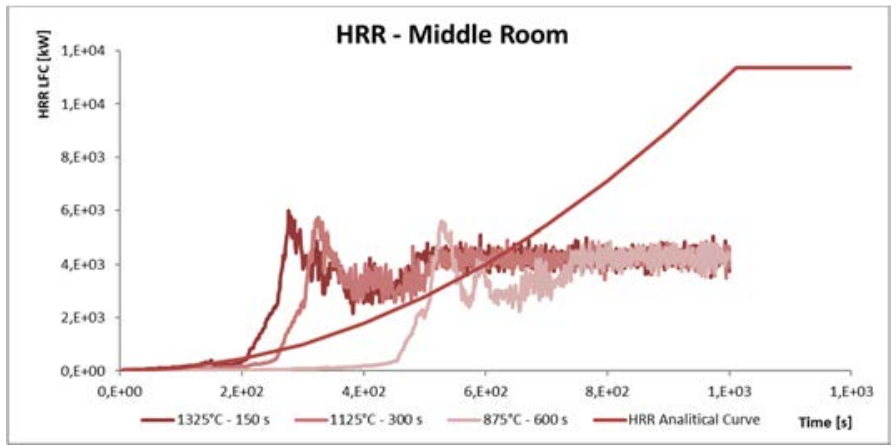

Figure 8: HRR(t) likely curve simulated in middle room with FDS5.

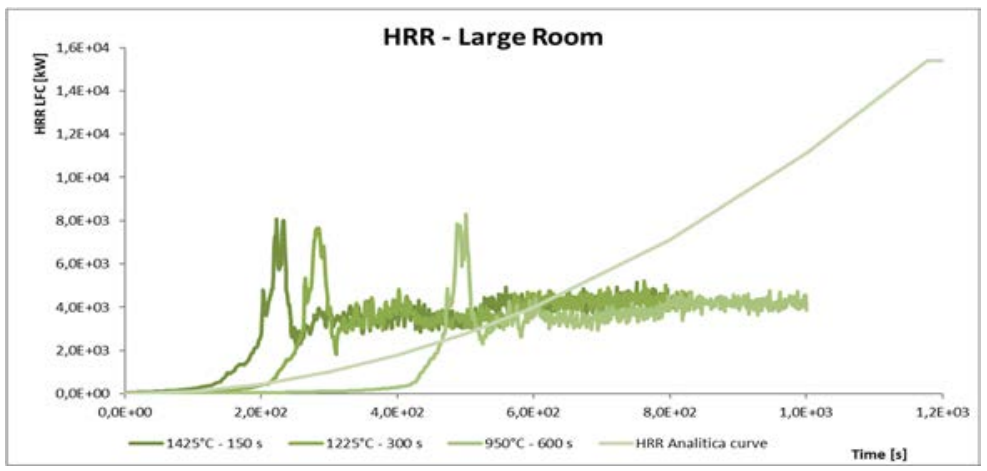

Figure 9: HRR(t) likely curve simulated in large room with FDS5. 


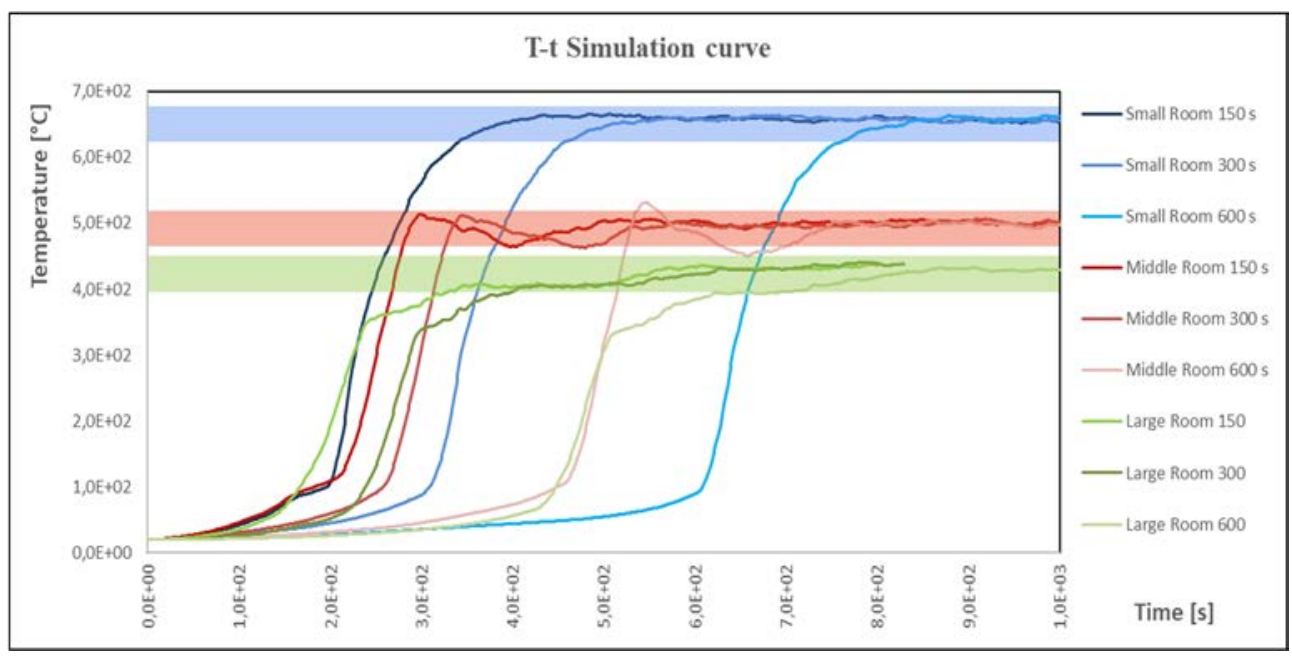

Figure 10: Temperature - time curve simulated in FDS5 [device 8].

The performance of the $\mathrm{HRR}_{\mathrm{LFC}}$ functions shows an interesting correlation in the behaviour of combustible materials present in the three simulated multi-task rooms at different moments described below:

- The rising phase that is strongly correlated with the trigger characteristic;

- The development phase until the achievement of a thermal peak power, with the overcoming of the flashover point;

- A phase of decrease due to the extinction of the portion of fuel initially triggered, and a final phase from when the fire reaches a plateau until the end of simulation.

The HRR $\mathrm{LFC}_{\mathrm{L}}$ curve can be linearized, step by step, as an iso-integral function of the simulation curve. The main objective of the linearization lies in the use of the data set FDS EVAC as input for large-scale simulation.

The temperature trend in each room shows that the maximum values are recorded on the ceiling, due to the fact that hot gases normally layer up.

The maximum temperatures recorded by the probes on the ceiling show that the smallest room reaches the highest temperatures in the shortest time: this phenomenon is due to a different condition of hot gases dilution for the different dimensions of the windows. The temperatures recorded for this geometry after a growth phase reach a plateau at $650^{\circ} \mathrm{C}$, while for the middle room the plateau value is $500^{\circ} \mathrm{C}$ and for the large room is $400^{\circ} \mathrm{C}$. (See Fig. 10.)

6 COMPARISON AND CONCLUSION: HRR LFC $_{\text {VS HRR }}$ ANALYTCAL

The results obtained in the likely fire curve guarantee a good representation of the analyzed case.

The HRR $_{\text {Analycal }}$ always has highest thermal peak values even if they are achieved in a longer time than $\mathrm{HRR}_{\mathrm{LFC}}$.

1. Below the differences in thermodynamic behaviour of the two HRR functions are summarized: The performance of $H_{R R}$ Analytical compared to the initial phase of the 
likely fire curve generally shows the tendency to underestimate the hazard of the simulated phenomena in order to evaluate the safety condition for exposed persons. These criteria are valid until the intersection point of the curves.

2. The thermal power achieved by $H R R_{\mathrm{LFC}}$ in the plateau phase is constant in the nine simulations carried out, in contrast with the result of the analytic curves in which the maximum value of HRR is always increasing.

The reasons for this variation are implicit in the analytical model hypothesis, because:

1. The value of the flashover, according to the Thomas method, is based only on the planimetric characteristics of the room, while the maximum HRR is also based on the opening factor.

2. The combustible material present in the compartment is considered as a total energy load uniformly distributed on the area, without taking into account the real layout of the furniture or the relative distance between the trigger and the remaining combustible materials.

3. This fire design assessment method is adopted to represent a thermal stress condition for structural verification, while the method described with the HRRLFC underline a more pronounced hazard features in the ignition phase, that instead involve the activation of emergency and evacuation procedures.

In conclusion, particularly complex cases must envision a design mode able to see solutions to the problem of personal safety in a holistic framework.

Using only theoretical evaluations or experimental tests on partial samples, the thermalchemical model stress [12] appears to be necessary, but not sufficient for specific purpose and needs to be integrated by a case by case modelling.

\section{REFERENCES}

[1] D.P.R. 1 agosto 2011, n. 151, Regolamento recante semplificazione della disciplina dei procedimenti relativi alla prevenzione incendi.

[2] D.M. 16 luglio 2014 "Regola tecnica di prevenzione incendi per la progettazione, costruzione ed esercizio degli asili nido".

[3] D.M. 9 maggio 2007 "Direttive per l'attuazione dell'approccio ingegneristico alla sicurezza antincendio"

[4] McGrattan, K., Mcdermott, R., Hostikka, S. \& Floyd, J., Fire Dynamics Simulator (Version 5) Users Guide, Nist Spec. Publ., 5, p. 220, 2010.

[5] Forney, G.P., User's Guide for Smokeview Version 5 - A Tool for Visualizing Fire Dynamics Simulation Data, 1(5), 2007.

[6] Lombardi, M., Rossi, G., Sciarretta, N. \& Oranges, N., Fire Design: Direct Comparison Between Fire Curves. the Case Study of a Nursery," Am. J. Eng. Appl. Sci., 6(3), pp. 297-308, Mar. 2013.

[7] ISO/TR 16738:2009, Fire-safety engineering -- Technical information on methods for evaluating behaviour and movement of people, 2009.

[8] Wen, J.X., Kang, K., Donchev, T. \& Karwatzki, J.M., Validation of FDS for the prediction of medium-scale pool fires, Fire Saf. J., 42(2), pp. 127-138, 2007.

[9] Korhonen T., VTT, Fire Dynamics Simulator with Evacuation: FDS+Evac Technical Reference and User's Guide, 2014. 
130 Safety and Security Engineering VII

[10] Hansen, R., Methodologies for calculating the overall heat release rate of a vehicle in an underground structure.

[11] NFPA, NFPA 555: Guide on Methods for Evaluating Potential for Room Flashover, 2013.

[12] Babrauskas, V., Heat Release Rates, in SFPE Handbook of Fire Protection Engineering, New York, NY: Springer New York, pp. 799-904, 2016.

[13] EN 1991-1-2: Eurocode 1: Actions on structures - Part 1-2: General actions - Actions on structures exposed to fire, 2002.

[14] Jesus de la Quintana, Integrated Fire Engineering and Response (IFER).

[15] Systems, S.M. \& Spaces, L., Nfpa 92B, Smoke Manag. malls, atria large areas, 2005. 